\title{
THE
}

\section{Renormalization group and other calculations for the one-dimensional spin-1/2 dimerized Heisenberg antiferromagnet}

\author{
J. N. Fields \\ Hendrik W. J. Blöte \\ University of Rhode Island \\ Jill C. Bonner \\ University of Rhode Island
}

Follow this and additional works at: https://digitalcommons.uri.edu/phys_facpubs

Terms of Use

All rights reserved under copyright.

\section{Citation/Publisher Attribution}

Fields, J. N., Blöte, H. W. J., \& Bonner, J. C. (1979). Renormalization group and other calculations for the one-dimenaional spin-1/2 dimerized Heisenberg antiferromagnet. J. Appl. Phys., 50, 1807-1809. Available at: https://doi.org/10.1063/1.327176 


\section{A|P| $\left.\right|_{\text {Applied Physics }} ^{\substack{\text { Journal of } \\ \text { Ans }}}$}

\section{Renormalization group and other calculations for the one-dimensional spin-1/2 dimerized Heisenberg antiferromagnet}

J. N. Fields, H. W. J. Blöte, and J. C. Bonner

Citation: Journal of Applied Physics 50, 1807 (1979); doi: 10.1063/1.327176

View online: http://dx.doi.org/10.1063/1.327176

View Table of Contents: http://scitation.aip.org/content/aip/journal/jap/50/B3?ver=pdfcov

Published by the AIP Publishing

Articles you may be interested in

Dynamics of the one-dimensional spin-1 Heisenberg antiferromagnet with exchange and single-site anisotropy

J. Appl. Phys. 75, 5937 (1994); 10.1063/1.355519

Spin-spin correlations in the one-dimensional spin-1/2, antiferromagnetic Heisenberg chain

J. Appl. Phys. 69, 5947 (1991); 10.1063/1.347825

Spin flop in a one-dimensional Heisenberg antiferromagnet(a)

J. Appl. Phys. 50, 1664 (1979); 10.1063/1.327230

Observation of the dynamic behavior of the antiferromagnetic-ferromagnetic phase transition in the onedimensional spin-1/2 antiferromagnet $\alpha-C u N S a l$

J. Appl. Phys. 50, 1859 (1979); 10.1063/1.327145

The spectral weight of low-lying excitations in the one-dimensional spin 1/2 Heisenberg antiferromagnet AIP Conf. Proc. 24, 335 (1975); 10.1063/1.30115

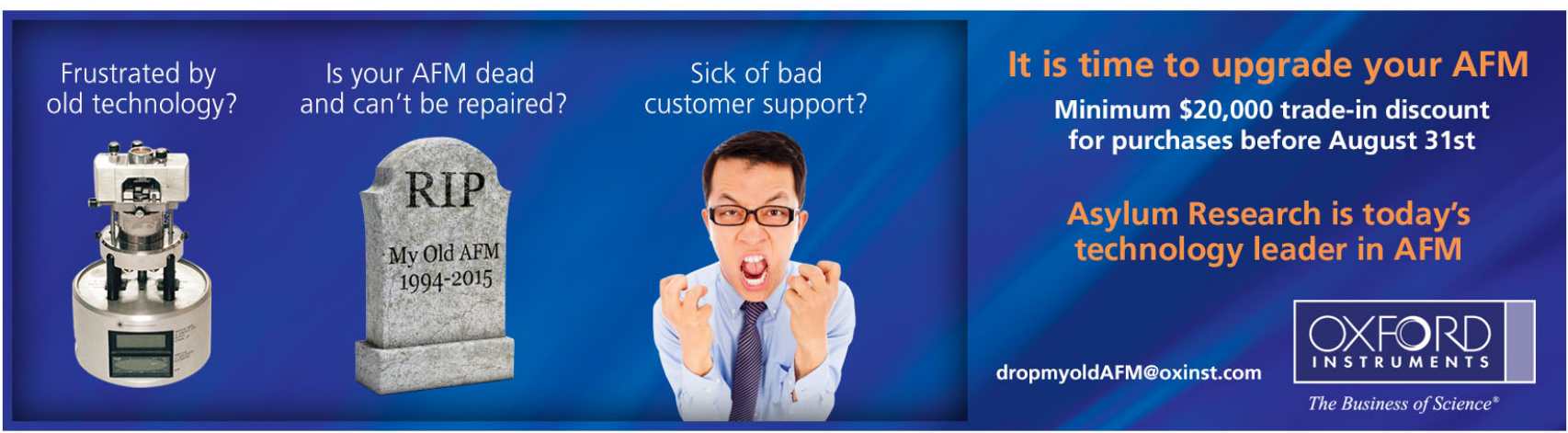




\title{
Renormalization group and other calculations for the one- dimensional spin-1/2 dimerized Heisenberg antiferromagnet ${ }^{(a)}$
}

\author{
J. N. Fields ${ }^{(b)}$ \\ Brookhaven National Lab., Upton, L.I., New York 11973 \\ H. W. J. Blöte and J. C. Bonner(c) \\ Physics Department, University of Rhode Island, Kingston, Rhode Island 02881
}

\begin{abstract}
A zero-temperature renormalization group (RG) approach is applied to the one-dimensional, spin-1/2 antiferromagnetic Heisenberg dimerized (alternating) chain. Specifically, the ground state energy and lowest-lying spectral excitations are examined. The calculation indicates the existence of a gap in the spectrum of the dimerized chain which vanishes only in the limit of a uniform spin chain, in contrast to a recent Green's function approach. The RG results are in reasonable agreement with numerical extrapolations on the exact eigenvalue spectrum of finite chains of up to 12 spins. Both methods are compared with several other approximate treatments of the Heisenberg system, and tested by comparison with exact results for the spin- $1 / 2 \mathrm{XY}$ dimerized chain.
\end{abstract}

PACS numbers: $75.10 . \mathrm{Jm}, 75.40 . \mathrm{Fa}$

\section{INTRODUCTION}

Recent experimental studies of linear chain spinPeierls systems such as TTF $\mathrm{CuS}_{4}\left(\mathrm{CF}_{3}\right)_{4}$ have been interpreted $[1,2]$ in terms of a Hartree-Fock (HFA) treatment [3] of the dimerized spin-1/2 anti-ferromagnetic Heisenberg chain, whose Hamiltonian is

$$
\text { if }=2 \sum_{i=1}^{N}\left[J_{1} \vec{s}_{2 i} \cdot \vec{s}_{2 i+1}+J_{2} \vec{s}_{2 i+1} \cdot \vec{s}_{2 i+2}\right\}
$$

where we take $J_{1} \geq J_{2}>0$. To the extent that this interpretation depends upon the existence of a gap in the dimerized Heisenberg system for all $J_{2}<J_{1}$, it has been called into question by a recent calculation [4] based on a Green's function decoupling scheme, which produces a gapless excitation band.

In this paper we discuss and compare two very recent approximate calculations of the ground state energy and low-lying excitations of tiamiltonian (1). The first calculation, which we shall present in some detail, is a zero-temperature quantum renormalization group ( $R G$ ) treatment based on a simple two-level truncation scheme [5]. However, since the dimer spectrum has a four-level (singlet-triplet) rather than a two-level character, a four-level quantum RG scheme which preserves the basic singlet-triplet character, has also been employed, to increase quantitative accuracy [7].

The second method consists of direct extrapolations on the exact eigenvalue spectrum of finite chains of up to 12 spins [6].

Finally the various results have been compared with several other approximate calculations.

\section{THE RENORMALIZATION GROUP METHOD}

In this section we describe calculations which are similar in approach to the lattice RG techniques frequent1y used to calculate critical behavior [8]. We shall establish recursion relations which define the Hamiltonian, and we shall find fixed-points of these recursion relations.

In this approach, the lattice is sub-divided into coupled blocks of $\mathrm{N}_{\mathrm{s}}$ sites such that the efgenvalues

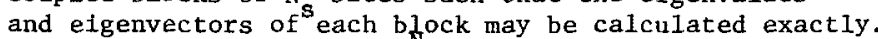
The basis of each block ( $2^{N_{S}}$ levels) is truncated to some number, $\mathrm{N}_{L}$, of levels, and the coupling between adjacent blocks is written within the truncated basis. The blocks have thus become equivalent to single sites on a new lattice. Choosing the two lowest states of an odd $-\mathrm{N}_{\mathrm{S}}$ block, for example, maps the block onto a single spin $1 / 2$ in the new lattice, and allows us to write down explicitly a set of recursion relations which define an RG transformation for the ground state of the Hamiltonian, at least for small $\mathrm{N}_{\mathrm{s}}$.

In order to maintain the symetries of the system throughout the iterative process, we cast the Hamiltonian into the form

$$
\begin{aligned}
H^{(n)}= & J^{(n)} \sum_{i}\left\{\left(S_{2 i}^{+} S_{2 i+1}^{-}+S_{2 i}^{-} S_{2 i+1}^{+}\right)+2 \mu{ }^{(n)} S_{2 i}^{z} s_{2 i+1}^{z}\right) \\
& +\alpha^{(n)}\left(S_{2 i+1}^{+} S_{2 i+2}^{-}+S_{2 i+1}^{-} S_{2 i+2}^{+}+2 v{ }^{(n)} S_{2 i+1}^{z} S_{2 i+2}^{z}\right) \\
& \left.+C^{(n)}\left(I_{2 i}+I_{2 i+1}\right)\right\}
\end{aligned}
$$

where I represents the $2 \times 2$ identity matrix. The initial conditions are

$$
\begin{gathered}
\mathrm{J}^{(0)}=\mathrm{J}_{1}, \mu^{(0)}=v^{(0)}=1, \alpha(0)=\alpha, \mathrm{C}^{(0)}=0, \\
\text { where } \alpha=J_{2} / \mathrm{J}_{1} .
\end{gathered}
$$

Associating the lattice sites into blocks of $\mathrm{N}_{\mathrm{S}}$ spins, the Hamiltonian can be written as a sum of intrablock and interblock terms:

$$
\begin{aligned}
H^{(n)} & =\sum_{p}\left\{H_{2 p}^{(n)}+H_{2 p+1}^{(n)}+v_{2 p, 2 p+1}^{(n)}+v_{2 p+1,2 p+2}^{(n)}\right. \\
& \left.\left.+C^{(n)}{ }_{i=1}^{N_{S}}{ }^{\Sigma_{I}} I_{2 p, i}+I_{2 p+1, i}\right)\right\}
\end{aligned}
$$

where $\mathbf{i}=1,2, \ldots, \mathrm{N}_{\mathrm{s}}$ labels the position of the site within block $2 \mathrm{p}$ or $2 \mathrm{p}+1$.

The intrablock terms have the form ( $\mathrm{N}_{S}$ odd)

$H_{2 p}=2 J\left\{\vec{s}_{2 p, 1} \cdot \vec{s}_{2 p, 2}+\alpha \vec{s}_{2 p, 2} \cdot \vec{s}_{2 p, 3}+\ldots+\alpha \vec{s}_{2 p, N_{s}-1} \cdot \vec{s}_{2 p, N_{S}}\right\}$

$H_{2 p+1}=2 J\left\{\alpha \vec{S}_{2 p+1,1} \cdot \overrightarrow{\mathrm{s}}_{2 \mathrm{p}+1,2}+\overrightarrow{\mathrm{S}}_{2 \mathrm{p}+1,2} \cdot \overrightarrow{\mathrm{S}}_{2 \mathrm{p}+1,3}+\ldots+\overrightarrow{\mathrm{S}}_{2 \mathrm{p}+1, \mathrm{~N}_{\mathrm{s}}-1}\right.$

$\cdot \overrightarrow{\mathrm{s}}_{2 \mathrm{p}+1, \mathrm{~N}}$

and the interblock couplings have the form

$$
\mathrm{v}_{2 \mathrm{p}, 2 \mathrm{p}+1}=2 \overrightarrow{\mathrm{S}}_{2 \mathrm{p}, \mathrm{N}_{\mathrm{s}}} \cdot \overrightarrow{\mathrm{s}}_{2 \mathrm{p}+1,1}
$$




$$
\mathrm{v}_{2 \mathrm{p}+1,2 \mathrm{p}+2}=2 \alpha \mathrm{J} \overrightarrow{\mathrm{s}}_{2 \mathrm{p}+1, \mathrm{~N}_{\mathrm{S}}} \cdot \overrightarrow{\mathrm{s}}_{2 \mathrm{p}+2,1}
$$

In equations (5) and (6) the labels $n$ are suppressed.

The eigenstates of $\mathrm{H}_{\mathrm{q}}(\mathrm{n})$, where $\mathrm{q}=2 \mathrm{p}$ or $2 \mathrm{p}+1$, may be chosen to be simultaneous eigenstates of the total z-component spin operator of the block $q$ :

$$
\mathrm{S}_{\mathrm{q}}^{z}=\stackrel{\mathrm{N}}{\mathrm{N}} \mathrm{i}_{\mathrm{N}}^{\mathrm{s}} \mathrm{s}_{\mathrm{q}, \mathrm{i}}^{\mathrm{z}}
$$

The new basis is chosen to contain the two states $\left\{\left|+{ }_{q},\right| \rightarrow q_{q}\right\}$, where $\mid+>_{q}\left(\mid->_{q}\right)$ is the state of lowest energy $E_{+}{ }^{q}\left(E_{-}\right)$in the subspace $s_{q}^{2}=t_{2}\left(-\frac{1}{2}\right)$. These states are the lowest energy states of the old basis and may be expressed as

$$
\begin{aligned}
& \left| \pm>(n+1)=\varepsilon_{2 p} \cdots \varepsilon_{\varepsilon_{1}} \lambda_{N_{S}}{ }_{\varepsilon_{1}}^{ \pm(n)} \cdots \varepsilon_{N_{S}}\right| \varepsilon_{1} \cdots \varepsilon_{N_{S}}>(n) \\
& \left| \pm>\begin{array}{l}
(n+1) \\
2 p+1
\end{array}=\varepsilon_{\varepsilon_{I}} \cdots \varepsilon_{\varepsilon_{N_{S}}} \Lambda_{\varepsilon_{I}}^{ \pm(n)} \cdots \varepsilon_{N_{S}}\right| \varepsilon_{1} \cdots \varepsilon_{N_{S}}>(n)
\end{aligned}
$$

where $\Sigma_{\varepsilon_{Q}}$ represents a summation over the two spin states $\pm(n)$ at site $\ell$ of the block.

The next step is to rewrite the interblock coupling terms. We calculate the matrix elements of $\mathrm{s}(\mathrm{n})$ and $\mathrm{S}_{\mathrm{q}, \ell}^{\mathrm{z}(\mathrm{n})}$ in the new basis:

$$
S_{2 p, l}^{+(n)}=\xi_{\ell}^{(n)}\left(\begin{array}{l}
01 \\
00
\end{array}\right)=\xi_{\ell}^{(n)} S_{2 p}^{+(n+1)}
$$

and, similarly,

$$
\begin{gathered}
\mathrm{S}_{2 p+1, \ell}^{+(n)}=\xi_{N_{S}-\ell+1}^{(n)} S_{2 p+1}^{+(n+1)}, \\
s_{2 p, l}^{z(n)}=n_{\ell}^{(n)} S_{2 p}^{z(n+1)} \\
s_{2 p+1, l}^{z(n)}=n_{N_{s}-l+1}^{(n)} s_{2 p+1}^{z(n+1)}
\end{gathered}
$$

where

$$
\begin{aligned}
\xi_{\ell}^{(n)=} & \Sigma_{\varepsilon_{1}} \cdots \varepsilon_{\varepsilon_{\ell-1}} \Sigma_{\varepsilon_{\ell+1}} \cdots \Sigma_{\varepsilon_{N_{S}}} \lambda_{\varepsilon_{1}}^{+(n)} \cdots \varepsilon_{\ell-1},+, \varepsilon_{\ell+1} \cdots \varepsilon_{N_{S}} x \\
& \lambda_{\varepsilon_{1}}^{-(n)} \cdots \varepsilon_{\ell-1},-, \varepsilon_{\ell+1} \cdots \varepsilon_{N_{S}},
\end{aligned}
$$

and

$$
\eta_{\ell}^{(n)}=\Sigma_{\varepsilon_{1}} \cdots \Sigma_{\varepsilon_{\ell}} \ldots \Sigma_{\varepsilon_{N_{S}}} \varepsilon_{\ell}\left(\lambda_{\varepsilon_{1}}^{+(n)} \ldots \varepsilon_{\ell} \ldots \varepsilon_{N_{S}}\right)^{2} .
$$

The coupling terms become

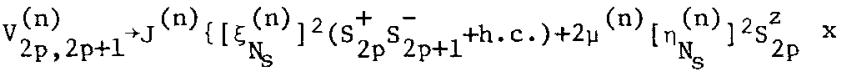

$$
\begin{aligned}
& \left.\mathrm{s}_{2 \mathrm{p}+1}^{\mathrm{z}}\right\}
\end{aligned}
$$

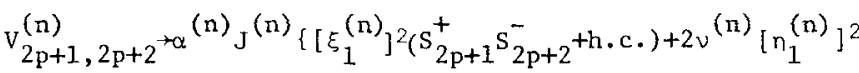

$$
\begin{aligned}
& x \mathrm{~s}_{2 \mathrm{p}+1}^{\mathrm{z}} \mathrm{s}_{2 \mathrm{p}+2}^{\mathrm{z}},
\end{aligned}
$$

and hence

$$
\begin{gathered}
J^{(n+1)}=\left[\xi_{N_{S}^{(n)}}^{(2)}\right]^{(n)} ; \quad a^{(n+1)}=\left[\xi_{1}^{(n)} / \xi_{N_{S}}^{(n)}\right]^{2}{ }^{(n)} ; \\
\mu^{(n+1)}=\left[n_{N_{S}}^{(n)} k_{N_{S}^{(n)}}^{(n)}\right]^{(n)} ; \nu^{(n+1)}=\left[n_{1}^{(n)} / \xi_{1}^{(n)}\right]^{2} v^{(n)} ; \\
C^{(n+1)}=N_{s} C^{(n)}+E_{+}^{(n+1)} .
\end{gathered}
$$

These recursion relations define an $\mathrm{RG}$ transformation for the ground state of the Haniltonian. The parameters $\mathrm{J}(\mathrm{n})$ and $\alpha^{(\mathrm{n})}$ provide information on the splittings of the lowest states of the system, $\mu(n)$ and $v(n)$ determine the symmetries of the fixed points, and $c^{(n)}$ may be used to find the ground state energy per site, $E_{0}$, through the relation

$$
E_{0}=\lim _{n \rightarrow \infty}\left(C(n) / N_{S}^{(n)}\right)
$$

In general, the eigenvectors $\mid \ddagger>(n+1)$ and eigenvalues $\mathrm{E}_{ \pm}^{(\mathrm{n}+1)}$, as well as the recursion coefficients of equation (12), are determined by machine.

RESULTS

The RG calculation has been performed for $N=3$, 5,7 and 9 , for both the Heisenberg and the XY dimer systems. The uniform Iimit, $a^{*}=1$, is always found to be an unstable fixed point of the system. Initial values $\alpha(0)<1$ flow (with increasing $n$ ) into the stable fixed point $\alpha^{*}=0$ (independent dimers). This result leads immediately to the conclusion that the alternating spectrum has a gap which vanishes only in the uniform limit $\alpha(0)=1$. This conclusion is supported by similar fixed point behavior in the case of the $X Y$ model, which is exactly solvable [9], and also shows a gap which vanishes only in the uniform limit. It is supported also by the direct finite chain extrapolations, which indicate a gap for both Heisenberg and XY dimerized systems $[5,6]$.

For both the ground state energy per spin (see Figs. 1 and 2) and the energy gap (see Fig. 3), the direct extrapolation method is believed to have the best quantitative accuracy for all degrees of dimerization. In Fig. 3, it is seen that the two-level RG method for $\mathrm{N}_{\mathrm{S}}=9$ (the accuracy of this method improves as $\mathrm{N}_{S}$ increases) is qualitatively in agreement with extrapolation results near the uniform limit, but is rather seriously in error near the dimer limit. This is the result of preserving only two levels at each it eration step. In order to improve the RG estimates for the gap (and also for the ground state energy per spin) another type of $R G$ calculation, in which the truncated basis at each step is taken to be four levels, preserving the singlet-triplet character, has been carried out, by way of a general matrix formulation of the problem which can be regarded as a variant on finite chain techniques $[10,11]$. The result is a considerable quantitative improvement for both gap and ground state energy. However, this method yields an $\alpha^{*}$ which is approximately rather than exactly unity.

The "critical exponents" for the Heisenberg problem are (a) the deviation of $\mathrm{E}_{\mathrm{o}} / \mathrm{J}$ [where $\mathrm{J}=\frac{1}{2}\left(\mathrm{~J}_{1}+\mathrm{J}_{2}\right)$ ] from the uniform limit as a function of $\delta[$ where $\delta=(1-\alpha)$ / $(1+a)]$, and (b) the vanishing of the gap $\Delta$ near the uniform limit, also as a function of $\delta$. A very recent theory by Cross and Fisher based on the dimerized Luther-Peschel-Luttinger model [12] predicts that the deviation (from $\left.\mathrm{E}_{\mathrm{O}}(\delta=0) / \mathrm{J}\right) \Sigma_{\mathrm{o}} / \mathrm{J} \sim \delta^{4} /{ }^{3}$, whereas an $\mathrm{N}_{\mathrm{S}}=3$, two-leve1 RG approach of de Braak et a1 [13] predicts $\varepsilon_{0} / \mathrm{J} \sim \delta^{1.37744}$

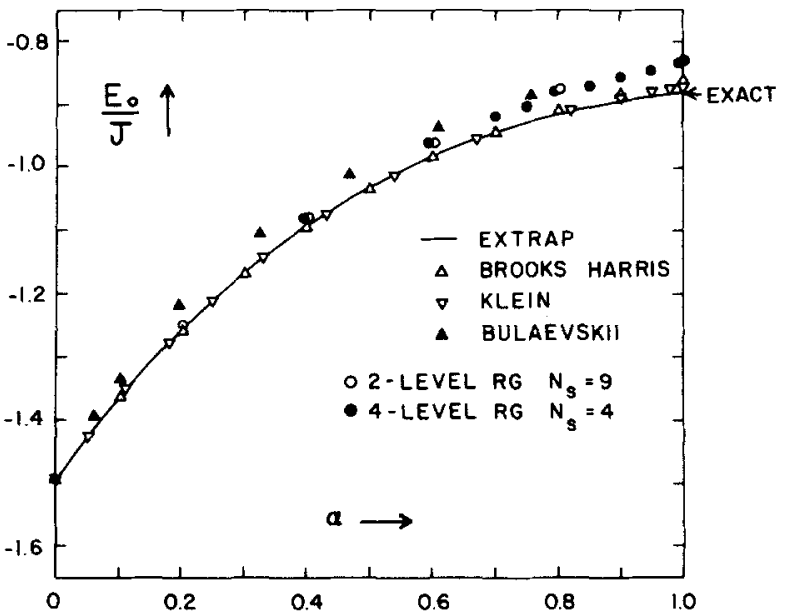

Fig. i. Comparison of ground state energy per spin for various models 


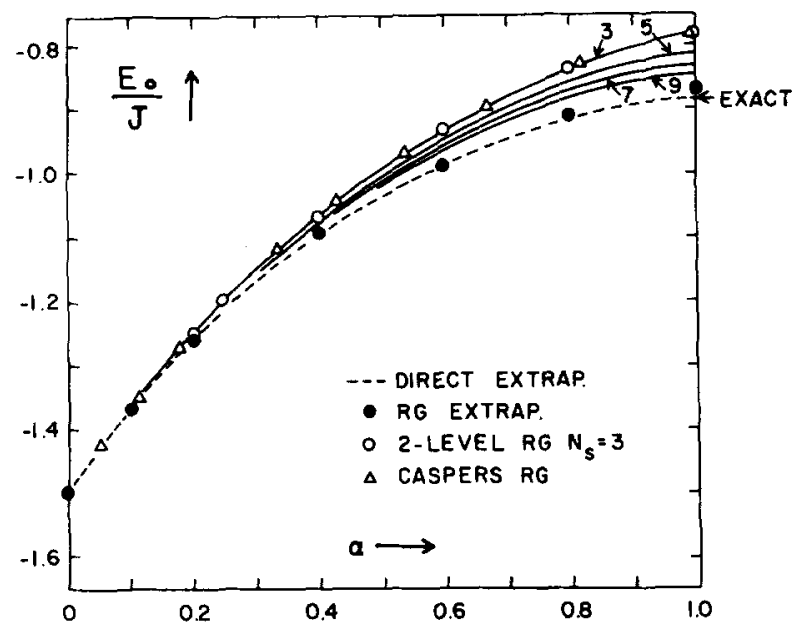

Fig. 2

Extrapolations of 2-level $R G$ groups for $\mathrm{N}_{\mathrm{s}}=3,5$, 7 , and 9 in comparison with direct extrapolations.

The finite chain extrapolations are consistent with either prediction, yielding an exponent $1.36_{-.02}^{+.01}$. The $\mathrm{N}_{\mathrm{s}}=7$, two-level RG approach, featured here gives a larger value of 1.5 (approx.). These four approaches are not consistent with the Bulaevskii HFA [3] which predicts $\mathcal{E}_{0} / \mathrm{J} \sim \delta^{2} \ell_{\mathrm{n}}^{2} \delta$.

For the case of the excitation energy gap the CrossFisher theory [12] predicts $\Delta / \mathrm{J} \sim \delta^{2 / 3}$, whereas our $\mathrm{N}_{\mathrm{s}}=7$ RG approach gives $\Delta / \mathrm{J} \delta^{0.76}$. (approx). These two ap proaches are in reasonable agreement. However, from Fig. 3, it appears that the finite chain extrapolations are predicting a somewhat larger exponent, but apparent$1 y<1$. Extrapolation uncertainties do not permit a quantitative estimate, however.

Finally, we discuss a varlety of other approximate calculations, In Fig. 1, the ground state energy per spin, $\mathrm{E} / \mathrm{J}$, is compared to the Bulaevskif $\mathrm{HFA}[3]$, and an appreclable quantitative discrepancy is observed for al1 $\alpha$. (The exact result [14], for the uniform limit only, is indicated by the arrow.) Much better agreement is achleved by a third-order perturbation calculation of Brooks Harris [15] and a Kekulé state calculation of Garcia-Bach and Klein [16]. It is interestlng that the Harris calculation is a perturbation about the dimer limit $(\alpha=0)$ and yet does quite well near $\alpha=1$. The 2-level RG result for $\mathrm{N}_{S}=9$ and the 4-level $\mathrm{RG}$ results for $\mathrm{N}_{\mathrm{s}}=4$ are intermediate in accuracy. However, In Fig. 2, the 2-level RG sequence for $\mathrm{N}_{s}=3,5,7$, and 9 is extrapolated and the result gives much better agreement with the direct extrapolations. (The $N=3$ RG calculation is equivalent to the RG calculation of ref. 13).

In the case of the energy gap (Fig. 3), the Bulaevskil HFA [3] shows the best agreement with the direct extrapolations. The Cross-Fisher calculation [12] is tnherently incapable of predicting amplitudes, and is therefore presented with arbitrary normalization. As mentioned above, the 2-leve1, $\mathrm{N}_{\mathrm{s}}$ odd, $\mathrm{RG}$ calculations are poor near the dimer 1imit. The 4-level, $N_{s}=4$, RG calculation with singlet-triplet symmetry is accurate $f$ $\alpha \leqslant 0.4$, but vanishes at $\alpha^{*}=0.962$ instead of $\alpha^{*}=1$. An older, quasi-boson calculation of Montgomery [17] gives a gap which vanishes as $(1-\alpha)^{\frac{1}{2}}$ or equivalently, $\Delta \sim \delta^{0.5}$.

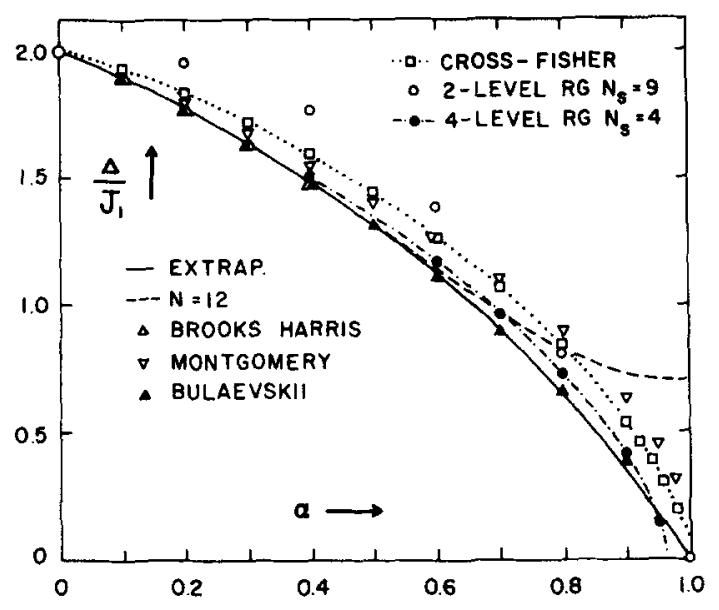

Fig. 3

Comparison of various approximate theories for the alternating antiferromagnetic excitation energy gap. The direct extrapolations are shown as a solid curve. As an aid to the eye, a dotted line has been drawn through the Cross-Fisher points and a dot-dashed Iine through the $\mathrm{N}_{\mathrm{s}}=4, \mathrm{~N}_{\mathrm{L}}=4 \mathrm{RG}$ points.

\section{REFERENCES}

a. Work supported by U.S.D.O.E. (for JNF) and by NATO and NSF grant no. DMR77-24136.

b. Now at Hughes Research Labs., Malibu, CA, 90265.

c. Fellow of the Institute for Independent 'Study, Radcliffe College.

1. J.W. Bray, et al., Phys. Rev. Lett. 35, 744 (1975).

2. I.S. Jacobs, J.W. Bray, H.R. Hart, Jr., L.V. Interrante, J.S. Kasper, G.D. Watkins, D.E. Prober, and J.C. Bonner, Phys. Rev. B14, 3036 (1976).

3. L.N. Bulaevskii, Sov. Phys. JETP 17, 684 (1963).

4. M. Drawid and J.W. Halley, AIP Conf. Proc. 34, 208 (1976).

5. J.N. Fields, to be published.

6. J.C. Bonner, J.D. Johnson and H.W.J. Blöte, J. Phys. (Paris), supplement, 39, C6-710 (1978). J.C. Bonner and H.W.J. Blöte. Unpublished work.

7. This approach is related to an $X Y$ model procedure in R. Julilen, J.N. Fields, and S. Doniach, Phys. Rev. Lett. 38, 1500 (1977); and Phys. Rev. B16, 4889 $(1977)$.

8. Th. Niemefjer and J.M.H. van Leeuwen, Phase Transitions and Critical Phenomena, edited by $C$. Domb and M.S. Green, Academic Press (London) Vol. 6 (1976).

9. P. Pincus, Solid state Commun. 91971 (1971); V.M. Kontorovich and V.M. Tsukernik, Sov. Phys. JETP 26, 687 (1968); J.H.H. Perk, H.W. Capel, M.J. Zuilhof, and Th. J. Siskens, Physica 81A, 319 (1975).

10. J.C. Bonner and M.E. Fisher, Phys. Rev. 135, A640 (1964).

11. W. Duffy, Jr., and K.P. Barr, Phys. Rev. 165, 647 (1968).

12. M. C. Cross and D.S. Fisher, to be published.

13. H. P. de Braak, W.J. Caspers, F.W. Wiegel, and M.W.M. Willemse, J. Stat. Phys. to be published.

14. L. Hulthén, Arkiv Mat. Astron. Fysik 26A, 1 (1938).

15. A.B. Harris, Phys. Rev. B7, 3166 (1973).

16. D.J. Klein and M.A. Garcia-Bach, Phys. Rev. B, to be published.

17. C.G. Montgomery, Ph.D. thesis, Cal. Tech. (1965). Unpublished. 